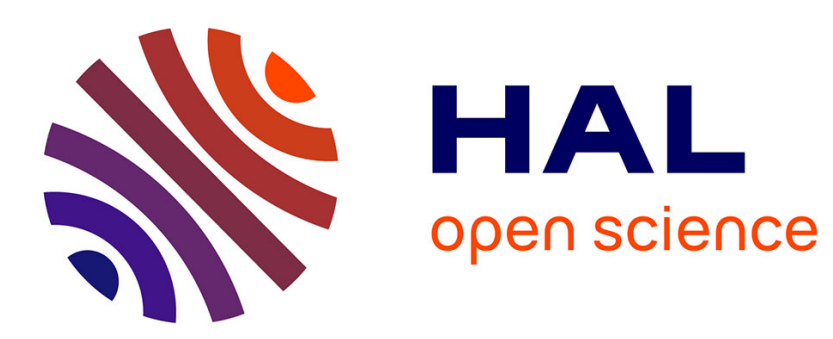

\title{
Temporal Interpolation Methods for Transient CHT
}

\author{
Rocco Moretti, Marc Errera, Frédéric Feyel
}

\section{To cite this version:}

Rocco Moretti, Marc Errera, Frédéric Feyel. Temporal Interpolation Methods for Transient CHT. AIAA Scitech 2019, Jan 2019, SAN DIEGO, United States. hal-02088826

\section{HAL Id: hal-02088826 https://hal.science/hal-02088826}

Submitted on 3 Apr 2019

HAL is a multi-disciplinary open access archive for the deposit and dissemination of scientific research documents, whether they are published or not. The documents may come from teaching and research institutions in France or abroad, or from public or private research centers.
L'archive ouverte pluridisciplinaire HAL, est destinée au dépôt et à la diffusion de documents scientifiques de niveau recherche, publiés ou non, émanant des établissements d'enseignement et de recherche français ou étrangers, des laboratoires publics ou privés. 


\title{
Temporal Interpolation Methods for Transient CHT
}

\author{
R. Moretti* and M. Errera ${ }^{\dagger}$ \\ DAAA, ONERA, Université Paris Saclay, Châtillon, France, 92322 \\ F. Feyel \\ SafranTech, Safran Group, Châteaufort, France, 78772
}

\begin{abstract}
The main objective of this paper is to present a methodology to carry out transient Conjugate Heat Transfer (CHT). Transient thermal interactions between a fluid and a solid are difficult to perform because of their computational cost. Indeed, fluid and solid characteristic times differ by several orders of magnitude. The present method has the aim to speed up the thermal analysis process by coupling an unsteady conduction computation with a sequence of steady CFD computations. This procedure assumes that the influence of fluid unsteadiness is negligible. Moreover, this paper proposes different approaches to interpolate fluid information between two steady CFD computations necessary to the solid solver for moving forward in time. The results obtained with these different approaches are compared with a reference result.
\end{abstract}

\section{Introduction}

$\mathrm{T}_{3}$ HERMAL stress analysis is an essential step in the design process of many industrial systems. In fact, high temperature gradients can lead to catastrophic system failure if they are not taken into account during the design process. In the case of fluid-structure thermal interaction this step becomes difficult especially due to huge temporal disparities. Generally, in industry, heat transfer analysis between fluid and structure is carried out through a Finite Element Analysis (FEA) using boundary condition values obtained from experimental tests or originated from empirical equations. Some methods have been developed to eliminate many of these assumptions creating a branch of the thermal science, the conjugate heat transfer (CHT). The concept was formulated in the early 1960's by Perelman [1]. Two approaches are mainly used for solving CHT problems: the monolithic and the partitioned approaches. The first one consists in solving fluid and structure problems with an unique system of equations (see Ref. [2]). This is a robust method but it is expensive and often difficult to implement. Moreover, it needs a specific solver able to compute fluid and solid problems together. The second approach, the partitioned one, consists of solving fluid and structure separately, and exchanging information with a specific frequency, as explained in [3]. This method is faster and more flexible than the monolithic one because it allows the use of an appropriate solver for each subproblem which means that different time steps can be used to speed up the resolution of each subproblem. Unfortunately, as the fluid-structure interface is not directly solved in the equation system, instability phenomena can occur. Many studies have been devoted to the stability of steady CHT problems (see Refs. [4-6]). The present paper focuses on the transient thermal interaction between fluid and structure. Unsteady CHT calculations are rather rare in practice because of their computational cost. In theory, to capture the transient thermal interaction, it is necessary to give to the solid solver information concerning the dynamics of the fluid. Fluid and structure should be coupled at each fluid time step, causing a very expensive solution, especially over long integration times, such as a full flight cycle. Furthermore, most of the time, only the thermal transient in the solid is sought. For this reason, a method that couples an unsteady computation in the solid with a sequence of steady state solutions in the fluid is generally used [7]. Most papers present an application of this method on aeronautic systems, high-pressure turbines in particular [8, 9], or a study on the stability of the coupling method [10], but the accuracy of this method has not been tested yet. This is the subject of the present paper. In the first part the "quasi-dynamic" coupling procedure is presented, then some different methods to improve its precision are proposed. In the last part a comparison of these methods is shown.

\footnotetext{
*Ph.D. Student, DAAA, ONERA, Université Paris Saclay, Mines ParisTech, rocco.moretti@onera.fr

${ }^{\dagger}$ Research Scientist, DAAA, ONERA, Université Paris Saclay, marc.errera@onera.fr

¥Professor, Safran Group, Université Paris Saclay, Mines ParisTech, frederic.feyel@ safrangroup.com
} 


\section{Methodology}

\section{A. Fluid-Solid Interfacial Conditions}

In CHT analysis it is common practice to impose a temperature as boundary condition on the fluid side and a heat flux on the solid side. Many studies have demonstrated that using a Robin condition with a well-chosen coefficient makes the CHT analysis more stable and faster than simply imposing the heat flux [6, 11]. Therefore, in this paper the Robin boundary condition in the solid solver is used. This condition can be expressed as follows:

$$
\hat{Q_{s}}+\alpha_{f} \hat{T}_{s}=Q_{f}+\alpha_{f} T_{f}
$$

more commonly expressed in the following form:

$$
\hat{Q}_{s}=Q_{f}+\alpha_{f}\left(T_{f}-\hat{T}_{s}\right)
$$

where $Q$ is the heat flux, $T$ is the temperature, and $\alpha$ is the coupling coefficient. The subscripts $f$ and $s$ indicate the fluid and the solid respectively. The caret $\hat{\boldsymbol{c}}$ denotes the sought variables. The coupling coefficient $\alpha$ is a relaxation coefficient and, physically, it can be regarded as a local interface stiffness.

When the Robin condition is used in a Conventional Staggered Scheme (CSS) algorithm, it is easy to show that if the CSS algorithm converges, the solution $\left(Q_{s}=Q_{f}\right.$ and $\left.T_{s}=T_{f}\right)$ is independent of $\alpha_{f}$.

\section{B. Coupling algorithm}

The difficulty of carrying out efficient coupling computations studying the aerothermal interaction between the fluid and the solid is due to their different time-scale necessary to solve each physical problem. Typically, the fluid dynamic problem requires a much smaller temporal resolution than the structural one. Taking the fluid time step as the coupling time period may be extremely expensive and pointless because no change in the thermal solid problem will be observed in such small time period. This is why in this kind of analysis the "quasi-dynamic" coupling approach, also referred to as the "quasi-steady" assumption, is used. Indeed, it is aimed at achieving a full transient analysis within the shortest possible time. It is based on coupling an unsteady thermal analysis in the solid with a sequence of steady CFD computations. Since the fluid characteristic time is much smaller than the solid one, the fluid unsteadiness can be neglected over the characteristic time of the solid. This justifies the use of steady CFD computations. Furthermore, these two calculations are iterated over a specific time interval, denoted as the coupling time step, until convergence. This iterative process turns the method into a strong coupling approach because the continuity of heat flux and temperature is respected at convergence of the coupling time step (see Ref. [10]).

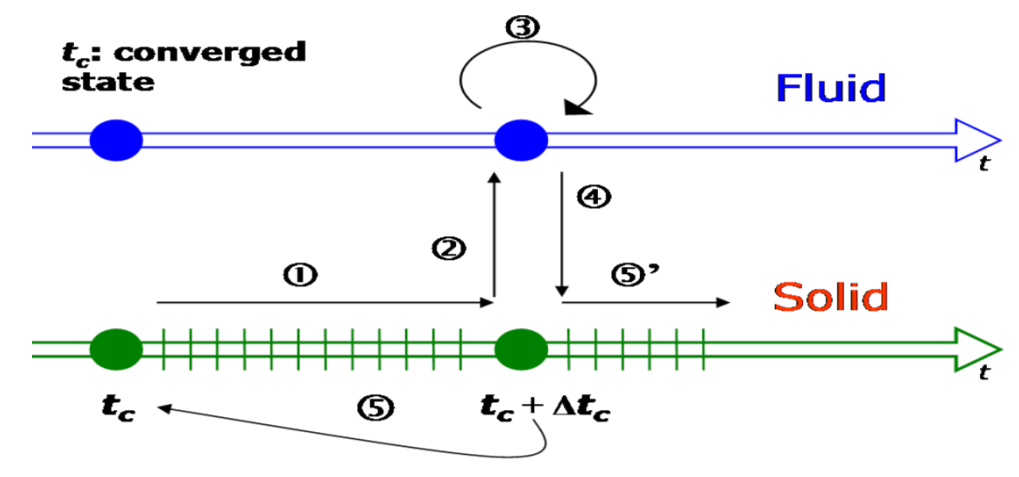

Fig. 1 Coupling algorithm for unsteady CHT problems. [10]

The various steps in Fig. 1 can be defined as follows:

(1) Transient calculation in the solid from $t_{c}$ to $t_{c}+\Delta t_{c}$

2 Exchange from the solid to the fluid solver

(3) Steady fluid computation 


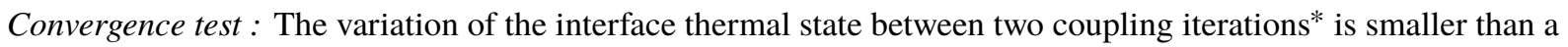
convergence criterion. Is the convergence attained?

(5) No: go back to $t_{c}$ (step (1)) and remake the coupling iteration

5. Yes: thermal transient state has been found, now moving forwards in time starting from $t_{c}+\Delta t_{c}$, that becomes the new converged state

The transient thermal state in the solid is calculated in step (1) using an interpolated fluid thermal state as boundary condition when the solid solution advances in time. This paper focuses on this part of the coupling algorithm.

\section{Boundary Condition Treatment for step one}

In the literature, it is usual to interpolate linearly in time both the fluid temperature and the fluid heat flux. We have tested different methods and we have compared them with a reference solution where neither interpolation nor sub-iterations are needed. The tested methods are the following:

- The Traditional Method (DR1): both fluid temperature and heat flux are interpolated linearly in time between two fluid values:

$$
\hat{Q}_{s}^{n}=\operatorname{inter}_{t}\left(Q_{f}^{n-1}\right)+\alpha_{f}\left(\text { inter }_{t}\left(T_{f}^{n-1}\right)-\hat{T}_{s}^{n}\right)
$$

where the superscript $n$ is the coupling iteration and $\operatorname{inter}_{x}(y)$ is the linear interpolation in respect of $x$ of the $y$ quantity.

- Interpolation in time (DR2): only the fluid heat flux is interpolated linearly in time. Indeed, the boundary condition on the fluid solver is a Dirichlet one, i.e. an imposed temperature condition, therefore the solid solver will receive theoretically the same temperature sent at the previous coupling iteration. Basically, the fluid and the solid temperatures are the same. This allows to avoid unnecessary spatial interpolation errors between the fluid and the solid meshes.

$$
\hat{Q}_{s}^{n}=\operatorname{inter}_{t}\left(Q_{f}^{n-1}\right)+\alpha_{f}\left(T_{s}^{n-1}-\hat{T}_{s}^{n}\right)
$$

- Heat Flux - Temperature Interpolation (DR3): here we use the solid temperature like in the previous method but in this case a linear interpolation between the fluid heat flux and the temperature is applied:

$$
\hat{Q}_{s}^{n}=\operatorname{inter}_{T_{s}^{n-1}}\left(Q_{f}^{n-1}\right)+\alpha_{f}\left(T_{s}^{n-1}-\hat{T}_{s}^{n}\right)
$$

This assumption is simply based on the Newton's law of Cooling:

$$
Q=h\left(T-T_{r e f}\right)
$$

where $h$ is the heat transfer coefficient and $T_{r e f}$ is a reference temperature.

As stated before, the results obtained using these three methods have been compared with a reference result. The latter has been obtained by coupling the fluid and the solid problems at every solid time step in order to have a solid Fourier number $D_{s}$ less or equal to 0.5 . Its expression is given by:

$$
D_{s}=\frac{a_{s} \Delta t_{s}}{\Delta y_{s}^{2}}
$$

where $a_{s}$ is the solid diffusivity $\left[\mathrm{m}^{2} / \mathrm{s}\right], \Delta t_{s}$ is the solid time step, and $\Delta y_{s}$ is the size of the first solid cell.

Because of the small Fourier number, sub-iterations are not necessary (step(5) over a coupling time step. Indeed, when the Fourier number is low the change of the thermal problem is very small, then no relaxation is needed consequently the error in the continuity of heat flux and temperature can be neglected.

An other important number in fluid-solid aerothemal analysis is the "mesh Biot number":

$$
B i^{4}=\frac{h}{k_{s} / \Delta y_{s}}
$$

\footnotetext{
*A coupling iteration contains algorithm steps from (1) to (4).
} 
where $h$ is the local heat transfer coefficient, $k_{s}$ is the solid conductivity and $\Delta y_{s}$ is the size of the first mesh of the solid domain. This number characterizes the heat transferred from the solid to the surrounding fluid, i.e. it gives an overview of the thermal fluid-structure interaction.

\section{Comparison of performance}

\section{A. Case Study}

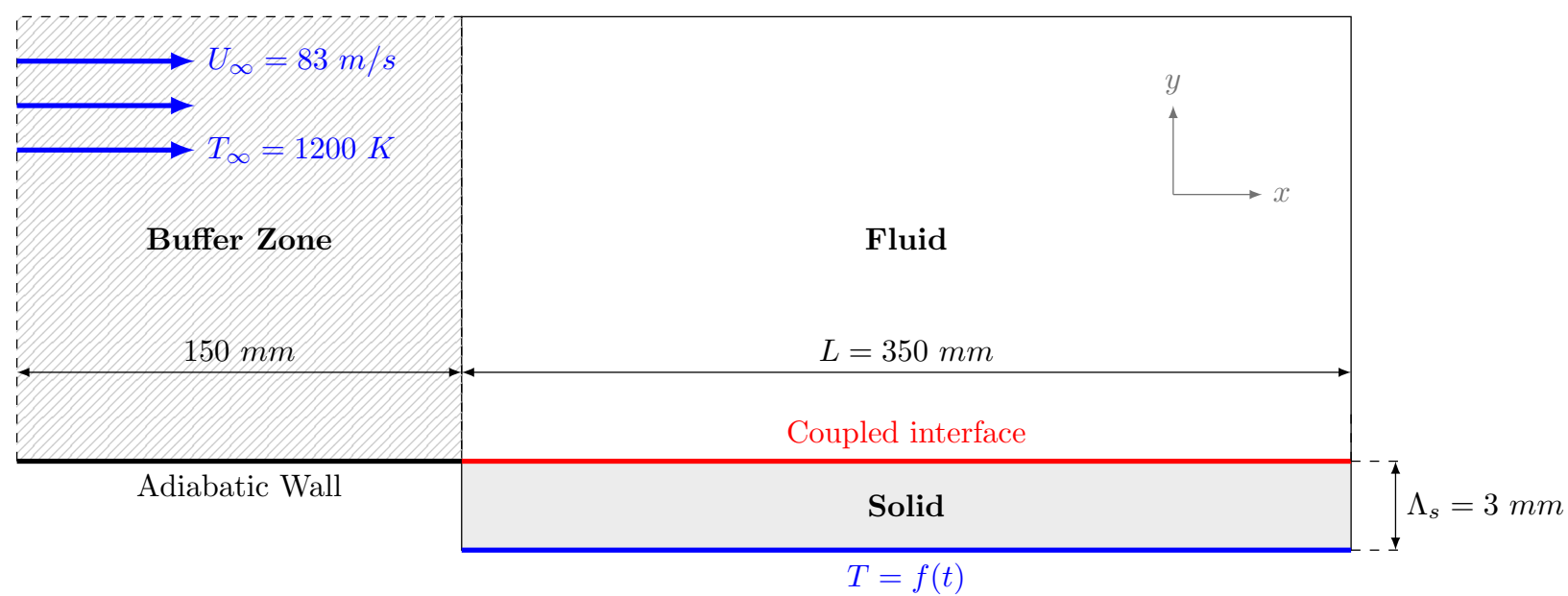

Fig. 2 Geometry and operating conditions.

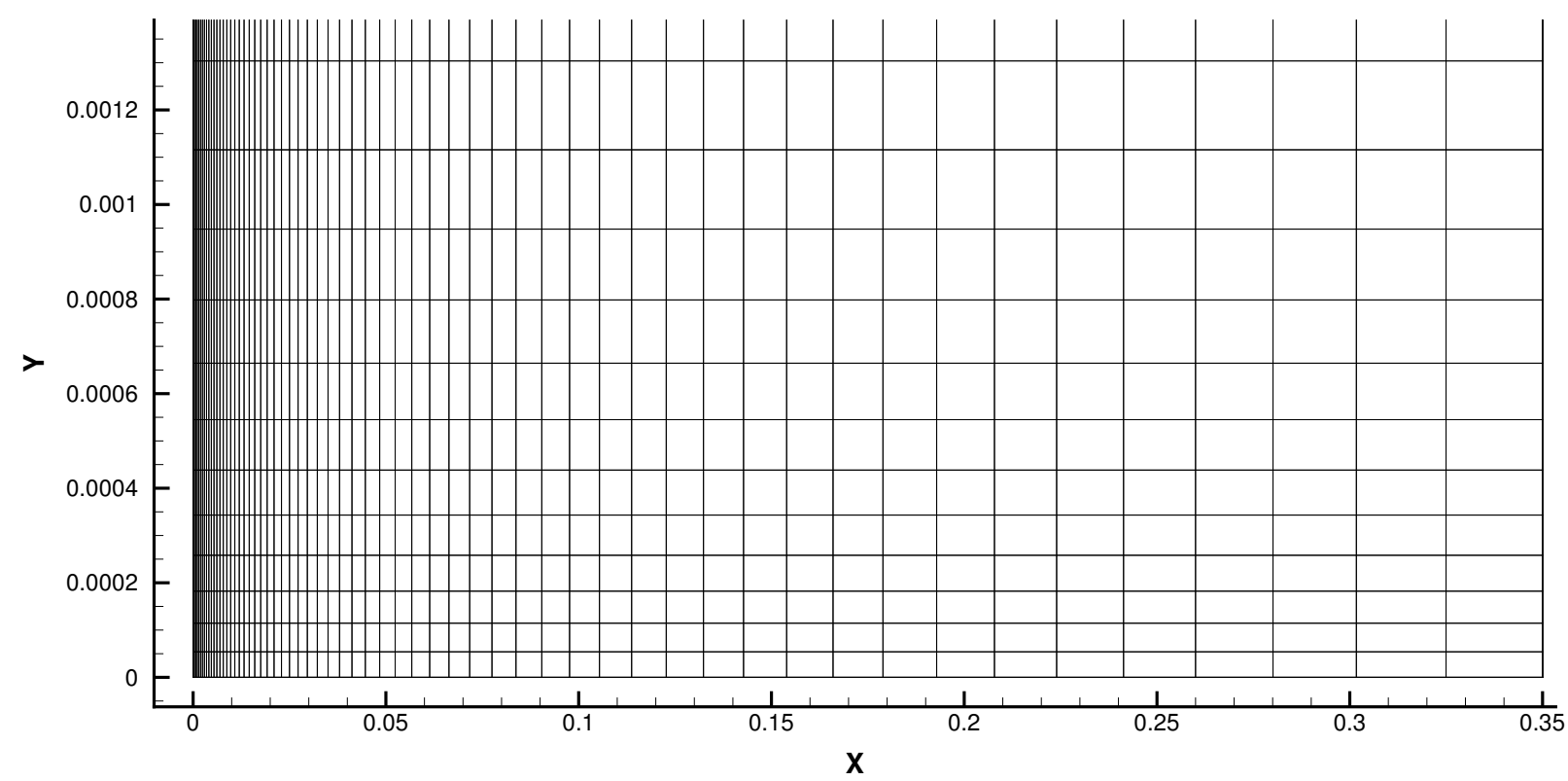

Fig. 3 Zoomed view of the fluid mesh near the leading edge $(x=0)$.

The different methods have been tested in a simple test case composed of convective heat transfer over, and solid conduction within a flat plate (see Fig. 2). In the same figure the main dimensions of the system and the main operating conditions are indicated. This test case might appear to be simple but from the thermal point of view is a complex case because the mesh near the leading edge has been refined enough in the flow direction to capture as much as possible the 


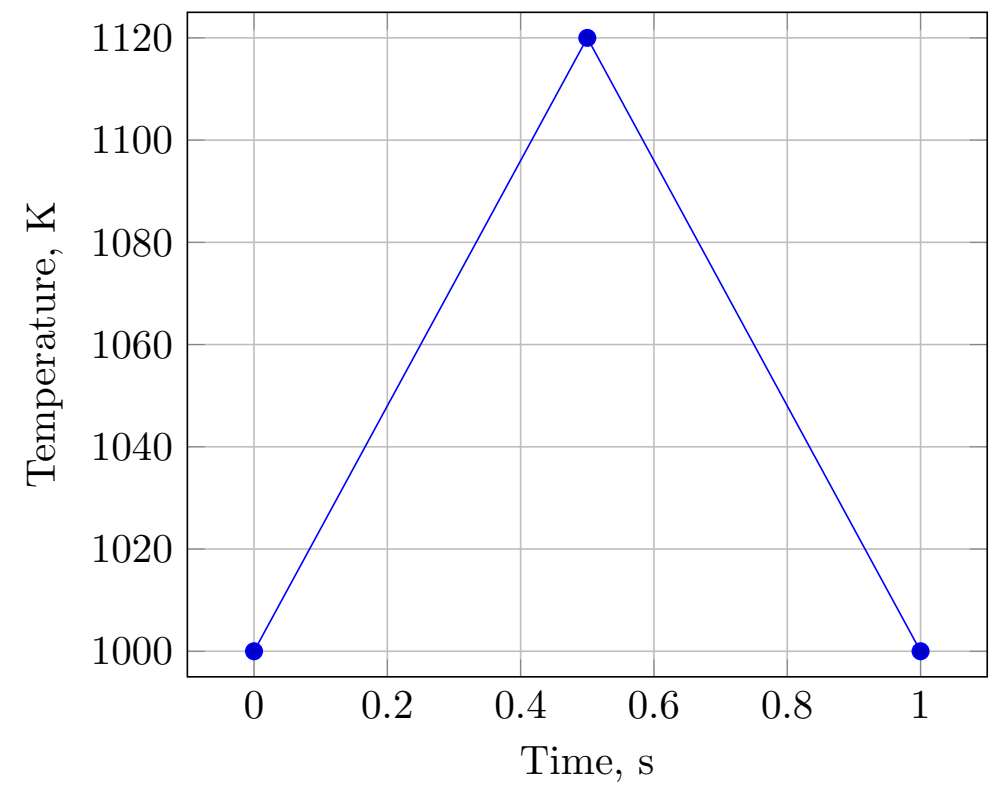

Fig. $4 T=f(t)$ imposed at the bottom face of the solid.

heat exchange (Fig. 3). Moreover, we have applied some severe operating conditions which lead to heat fluxes of the order of $10^{5} \mathrm{~W} / \mathrm{m}^{2}$. Indeed, the evolution in time of the temperature imposed on the bottom face of the solid, shown in Fig. 4, generates large temperature gradients in the solid (3 $\mathrm{mm}$ thick). The thermal properties used for the solid are: thermal conductivity of $13.6 \mathrm{~W} /(\mathrm{mK})$ and volumetric heat capacity of $1.36 \mathrm{~W} /\left(\mathrm{m}^{3} \mathrm{~K}\right)$. The three points in Fig. 4 indicate the time at which the solid solver is coupled with the fluid one. Between them the solid solver advances in time with interpolated values of the fluid states as boundary condition for the coupling interface. In this test case we have simulated the heat transfer between fluid and solid within a relatively short time (1 second) because of the computational time necessary to calculate the reference solution.

The software used to solve the fluid problem is elsA [12], whereas the unsteady conduction problem in the solid is solved by Z-set [13]. The connection between this two solvers is provided by using the coupling library CWIPI [14].

\section{B. Results}

Figure 5 shows the temperature evolution at the leading edge for two different values of the coupling coefficient $\alpha_{f}$ using the DR1 method. We can see that the two results are not identical, although they should be. In fact, as mentioned before, the solution should be independent of $\alpha_{f}$ at convergence. This discrepancy is due to the simultaneous use of the linear interpolation of the fluid temperature and of a large value of the coupling coefficient in the Robin formulation (see Eq. (1)). Indeed, the second condition means $\hat{T}_{s}=T_{f}$ that, together with the first one, produces a linear temperature.

The linear interpolation of the fluid temperature can be avoided when a Dirichlet condition is used on the fluid side of the coupled interface because this quantity is already known. In fact, a Dirichlet condition on the fluid side means $T_{f}^{n-1}=T_{s}^{n-1}$ and $T_{s}^{n}=T_{f}^{n-1}$, so $T_{s}^{n}=T_{s}^{n-1}$. The symbol $n$ in the previous equations indicates the current coupling iteration. Moreover, the fluid and the solid meshes are rarely coincident, Thus, the fluid and the solid temperatures may be pretty different due to interpolation errors.

Therefore, we have implemented the DR2 method, which uses the temperature computed by the solid solver at the previous coupling iteration instead of using the linear interpolation of the fluid temperature.

Figure 6 shows the temperature evolution using the DR2 method for the same two coefficients. In this case, the two results are identical, i.e. the $\alpha_{f}$ dependency has been removed. The DR3 method uses the same temperature of the DR2 method, therefore its $\alpha_{f}$ independence is automatically verified.

In view of these results, we have decided to exclude the DR1 method from the comparison with the reference solution.

Figure $7 \mathrm{a}$ shows this comparison of temperature. The DR3 method behaves like the reference calculation, whereas the temperature computed by the DR2 method is slightly different in the interpolated zone between two coupling points 


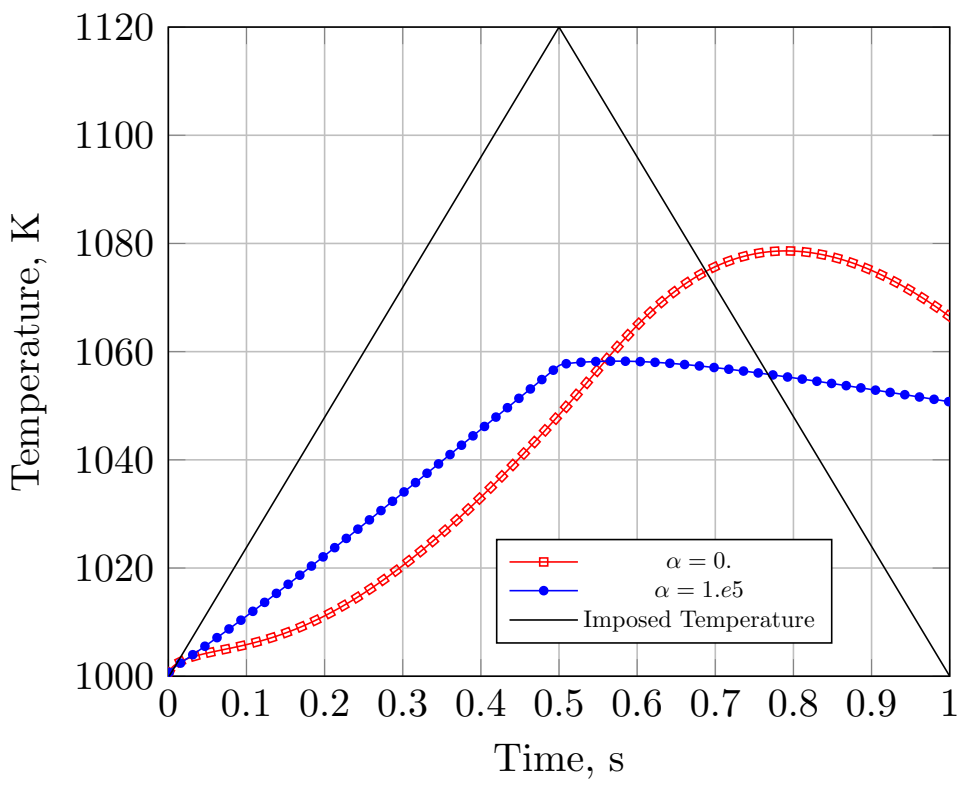

Fig. 5 Temperature result obtained with the DR1 method.

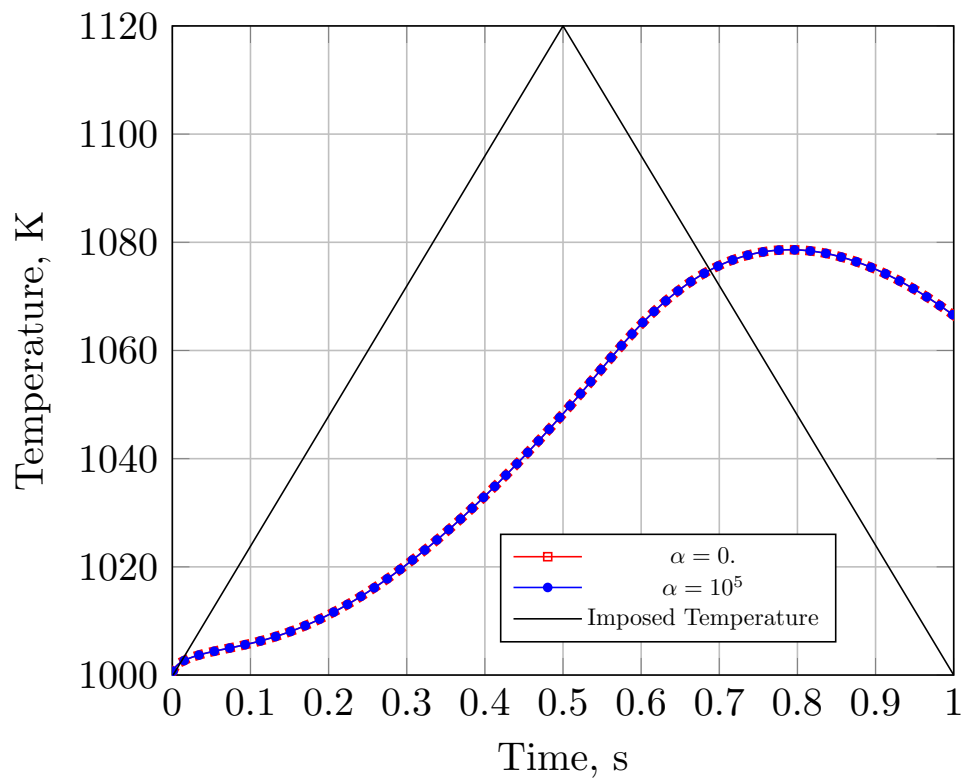

Fig. 6 Temperature result obtained by the DR2 method. 


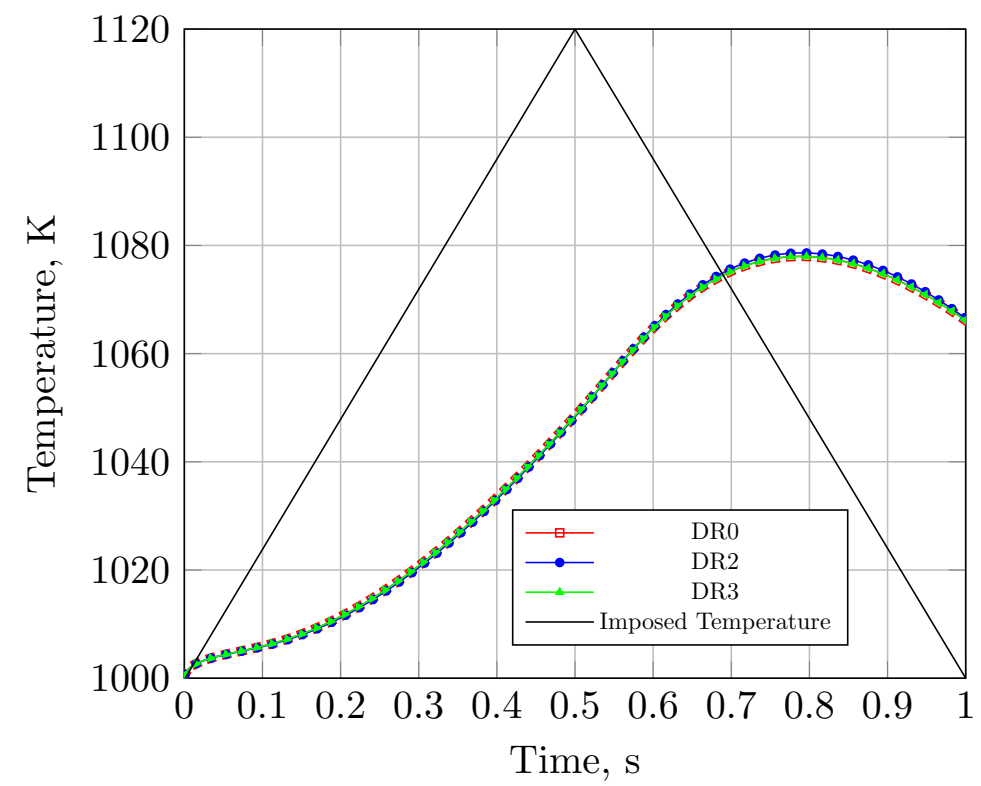

(a) Entire calculation.

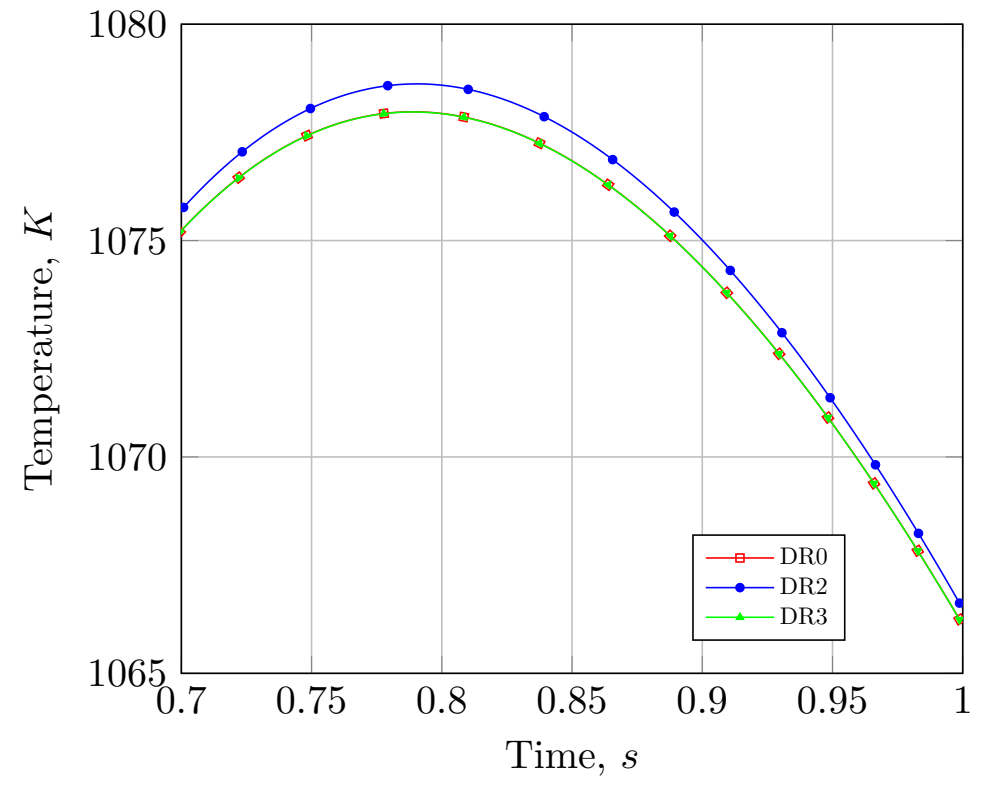

(b) Zoom view on the last part of the calculation.

Fig. 7 Comparison of DR2 and DR3 result with the reference solution for the temperature. 
(Fig. 7b). This difference is more evident on the heat flux at the leading edge (see Fig. 8). The DR2 heat flux evolution is almost linear because this method imposes a temporal linear heat flux, whereas the DR3 result compares well with the reference solution.

As a result, the temporal accuracy of the DR3 method allows us to obtain a precise solution of the heat load in the solid domain, not only at the coupling times, but also at each solid time step.

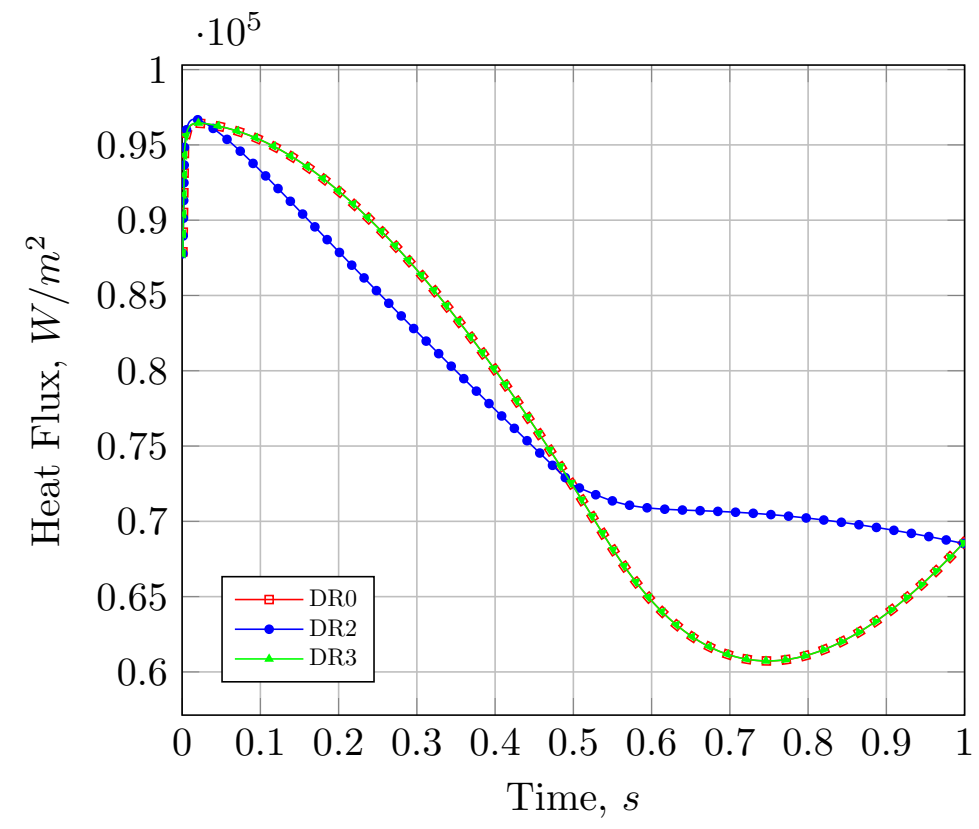

Fig. 8 Comparison of DR2 and DR3 result with the reference solution for the heat flux.

The same configuration with different thermal proprieties for the solid domain has been tested. In order to increase significantly the mesh Biot number, the solid conductivity $k_{s}$ has been changed from $13.6 \mathrm{~W} / \mathrm{mK}$ to $1.8 \mathrm{~W} / \mathrm{mK}$. Figures 9 and 10 compare the DR2 and DR3 methods for the temperature and the heat flux profiles. Figures 9a and $9 \mathrm{~b}$ shows the comparison of the temperature. In this case, the difference using the two methods is barely noticeable. Only zooming on the first part of the calculation (Figure 9b) it is possible to see a small deviation of the temperature profile computed by the DR2 method, whereas the DR3 method provides a profile that fits perfectly the reference solution. Looking at the heat flux solution in Figure 10, we notice only a small difference between the two solutions because the heat flux evolve approximately linearly in time. 


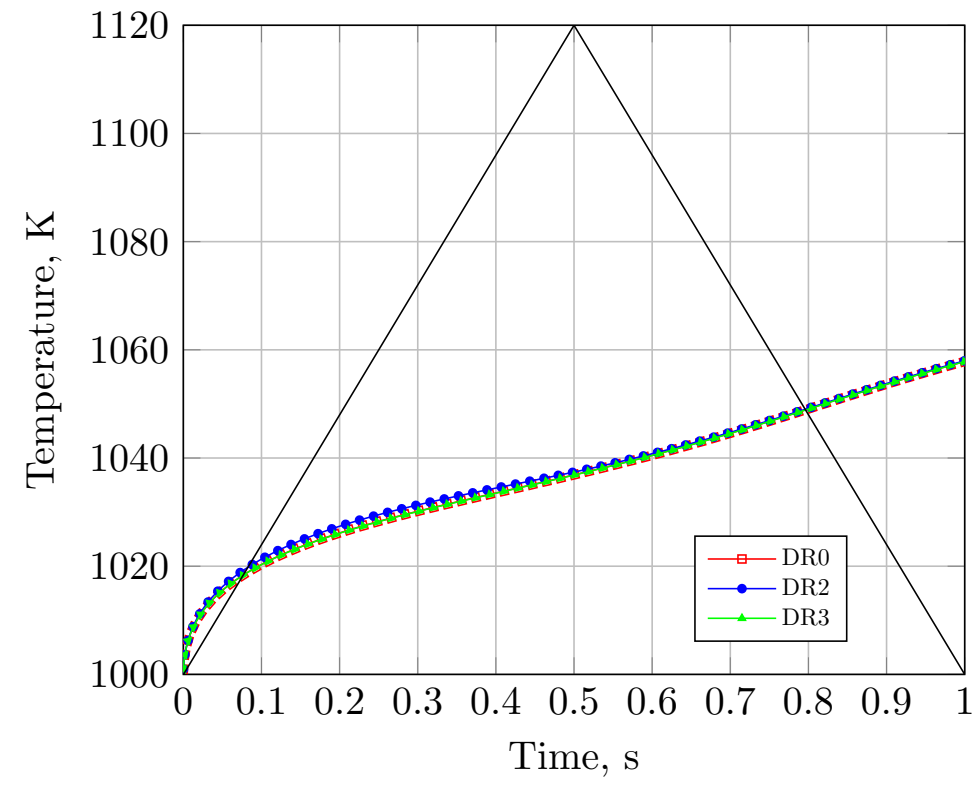

(a) Temperature profile on the entire computation.

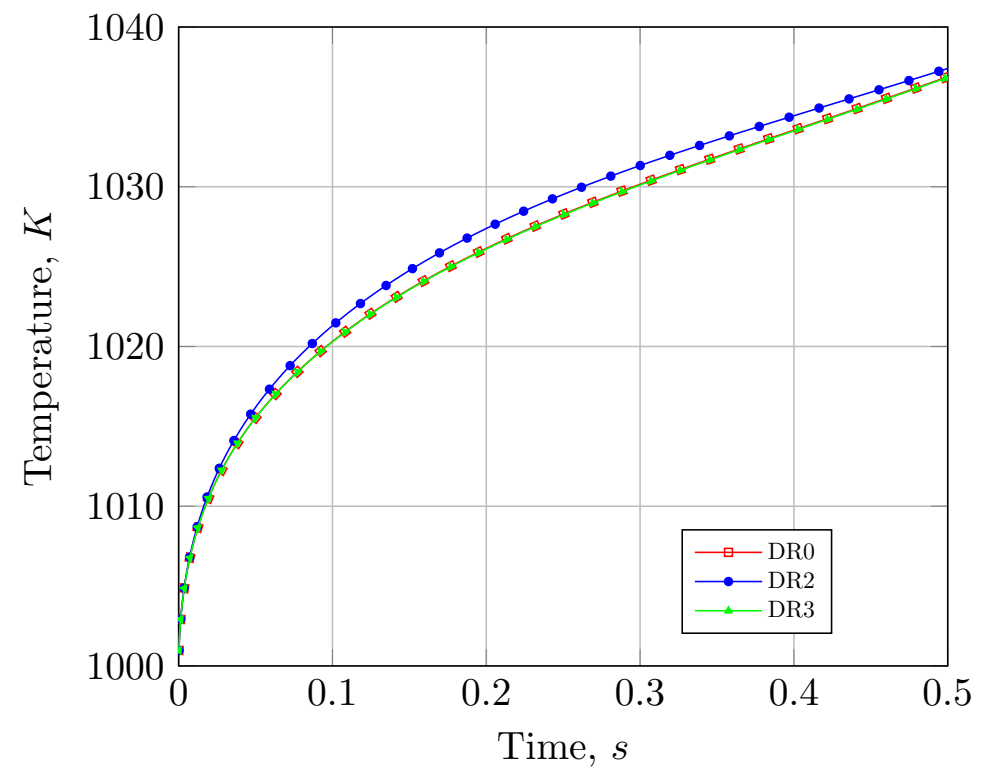

(b) Zoom view of the temperature profile on the first part of the computation.

Fig. 9 Comparison of DR2 and DR3 result with the reference solution for the temperature. 


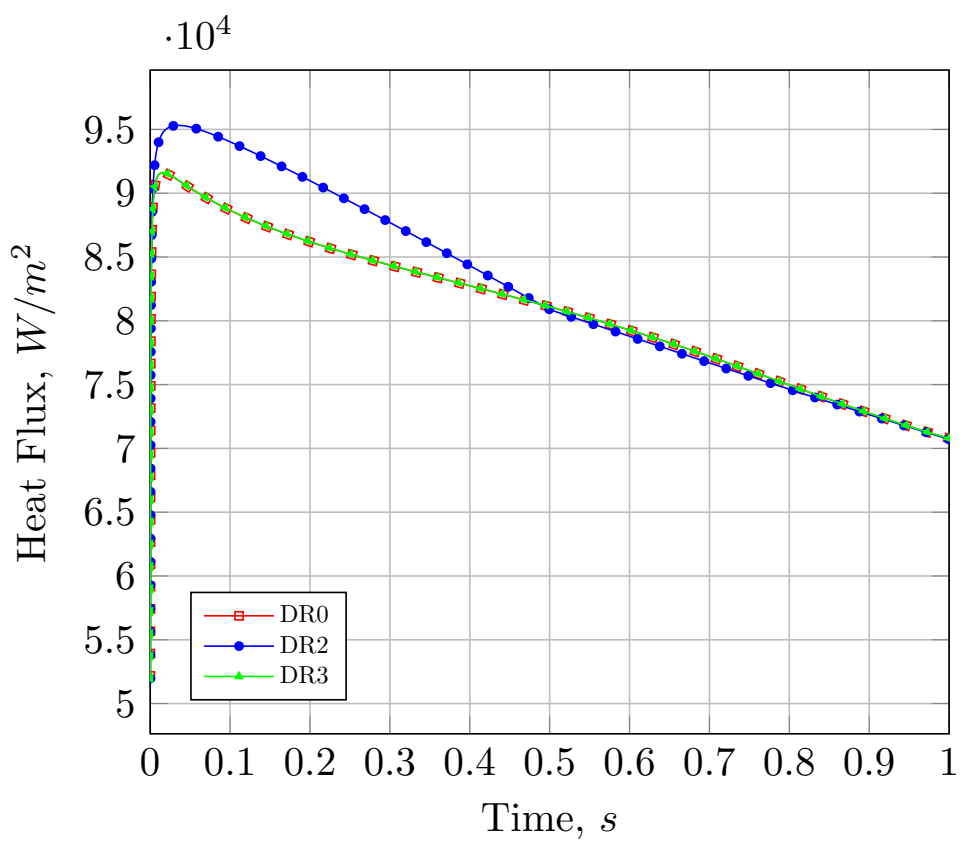

Fig. 10 Comparison of DR2 and DR3 result with the reference solution for the heat flux.

\section{Conclusion}

In this paper we have presented and compared different temporal interpolation methods in the framework of the "quasi-steady" assumption. We have shown that there are no physical reason to assume a linear variation in time of the heat flux (DR2). In fact, the heat flux is linearly dependent on the temperature (Eq. (6)), then an interpolation based on this hypothesis (DR3) is more appropriate. Indeed, the use of the heat flux-temperature linearity compares well with a reference solution based on a sub-iterative and unsteady procedure. It is a promising result because applying the DR3 method to the "quasi-dynamic" coupling algorithm, it is now possible to reduce the computational time cost of more than $95 \%$ while maintaining a very good accuracy.

\section{Acknowledgments}

The authors would like to acknowledge the Direction Scientifique Générale de l'ONERA for their support.

\section{References}

[1] Perelman, T., "On conjugated problems of heat transfer," International Journal of Heat and Mass Transfer, Vol. 3, No. 4, 1961, pp. 293-303. doi:10.1016/0017-9310(61)90044-8, URL http://www.sciencedirect.com/science/article/ pii/0017931061900448

[2] Malatip, A., Wansophark, N., and Dechaumphai, P., "Combined Streamline Upwind Petrov Galerkin method and segregated finite element algorithm for conjugate heat transfer problems," Journal of Mechanical Science and Technology, Vol. 20, No. 10, 2006, pp. 1741-1752. doi:10.1007/BF02916278, URL https://doi.org/10.1007/BF02916278.

[3] Felippa, C. A., and Park, K. C., "Staggered transient analysis procedures for coupled mechanical systems: formulation," Computer Methods in Applied Mechanics and Engineering, Vol. 24, No. 1, 1980, pp. 61-111. doi:https://doi.org/10.1016/00457825(80)90040-7.

[4] Giles, M. B., "Stability Analysis of numerical interface condition in fluid-structure thermal analysis," International Journal For Numerical Methods In Fluids, 1997, p. 421+436. 10.1002/(SICI)1097-0363(19970830)25:4<421::AID-FLD557>3.0.CO;2-J.

[5] Errera, M.-P., and Chemin, S., "Optimal solutions of numerical interface conditions in fluid-structure thermal analysis," Journal of Computational Physics, Vol. 245, No. Supplement C, 2013, pp. 431-455. doi:10.1016/j.jcp.2013.03.004, URL http://www.sciencedirect.com/science/article/pii/S0021999113001770 
[6] Moretti, R., Errera, M.-P., Couaillier, V., and Feyel, F., "Stability, convergence and optimization of interface treatments in weak and strong thermal fluid-structure interaction," International Journal of Thermal Sciences, Vol. 126, 2018, pp. 23-37. doi:10.1016/ j.ijthermalsci.2017.12.014, URL https://www.sciencedirect.com/science/article/pii/S1290072917308785

[7] Errera, M.-P., and Baqué, B., "A quasi-dynamic procedure for coupled thermal simulations," International Journal for Numerical Methods in Fluids, Vol. 72, No. 11, 2013, pp. 1183-1206. doi:10.1002/fld.3782, URL http://doi.wiley.com/10.1002/ fld. 3782

[8] Ganine, V., Javiya, U., Hills, N. J., and Chew, J., "Coupled Fluid-Structure Transient Thermal Analysis of a Gas Turbine Internal Air System With Multiple Cavities,” Journal of Engineering for Gas Turbines and Power, 2012.

[9] Sun, Z., Chew, J. W., Hills, N. J., Lewis, L., and Mabilat, C., "Coupled Aerothermomechanical Simulation for a Turbine Disk Through a Full Transient Cycle," Journal of Turbomachinery, 2012.

[10] Errera, M.-P., Lazareff, M., Garaud, J.-D., Soubrié, T., Douta, C., and Federici, T., "A coupling approach to modeling heat transfer during a full transient flight cycle," International Journal of Heat and Mass Transfer, Vol. 110, 2017, pp. 587-605. doi:10. 1016/j.ijheatmasstransfer.2017.03.048, URL http://linkinghub.elsevier.com/retrieve/pii/S0017931016331222

[11] Errera, M.-P., and Duchaine, F., "Comparative study of coupling coefficients in Dirichlet-Robin procedure for fluid-structure aerothermal simulations," Journal of Computational Physics, Vol. 312, No. Supplement C, 2016, pp. 218-234. doi: 10.1016/j.jcp.2016.02.022, URL http://www.sciencedirect.com/science/article/pii/S0021999116000760

[12] Cambier, L., and Gazaix, M., "elsA - An efficient object-oriented solution to CFD complexity," 40th AIAA Aerospace Sciences Meeting \& Exhibit, Aerospace Sciences Meetings, American Institute of Aeronautics and Astronautics, 2002. doi:10.2514/6.2002-108, URL https://doi .org/10.2514/6.2002-108.

[13] ParisTech, M., “ZeBuLoN Finite Element Code Z-set User Manual,”, 2012. URL http://www .zset-software.com/

[14] "La bibliothèque de couplage CWIPI | Coupling With Interpolation Parallel Interface," , 2000. URL https://w3 . onera.fr/ cwipi/. 